\title{
Rebound Property in Low Velocity Impact of Two Equivalent Balls
}

\author{
Eijiro Inamura*, Hirofumi Minamoto**, Shozo Kawamura*** \\ *(Tokyo Metropolitan College of Industrial Technology, Japan \\ Email: inamura@metro-cit.ac.jp) \\ ** (Department of Mechanical Engineering, Shizuoka Institute of Science and Technology, Japan \\ Email: minamoto.hirofumi@sist.ac.jp) \\ *** (Department of Mechanical Engineering, Toyohashi University of Technology, Japan \\ Email: kawamura@me.tut.ac.jp)
}

\begin{abstract}
The present study focuses on an impact phenomenon of two spheres and its rebound property. The collision of two spheres is a fundamental problem with impact phenomenon. The coefficient of restitution characterizes the property of impact phenomenon and has been estimated in general by experiment. In addition, it has been tried to estimate the coefficient by analytical and numerical methods. Considering body deformation, the body is deflected rapidly in collision and the strain rate occurred in the body is significantly high. It is well known the yield stress of the specific industrial material depends on the strain rate. However, it has not been proved the relationship between the coefficient of restitution and the strain rate in details yet. The present paper discusses the coefficient of restitution in low velocity impact of two equivalent balls. The impact experiments were conducted for the balls with different diameters by a pendulum impact apparatus. And the balls are numerically analyzed based on the finite element method considering the dependence of yield stress on strain rate as the material property of the balls. In conclusion, the strain rate decreases with the increment of the ball diameter and, it causes the coefficient of restitution to decrease.
\end{abstract}

Keywords: Coefficient of restitution, Elastic collision, Impact velocity, Inelastic collision, Size effect

\section{INTRODUCTION}

The behaviors of contact and collision of spheres are fundamental problems in mechanics of elasticity and machine dynamics, and so on. They have been interested from long ago [1]-[3] and investigated by using experimental, analytical and numerical methods. The outcomes of the investigations have been applied many objects in shot peening [4][5], sports engineering[6][7], automotive [8], and many others. The rebound properties are represented by coefficient of restitution, impact time and so on. They are changed with various causes such as ball size, material property, impact speed, and so on. Considering the causes, methodologies to obtain impact model and the coefficient of restitution have been improved until now [9][10]. Specifically, as a research about ball size effect, Aryaei et al. [11] fell balls 6 to12 $\mathrm{mm}$ in diameter on a plate at 0.7 to $3.2 \mathrm{~m} / \mathrm{s}$ speed. As the experimental and numerical results, they pointed out that the coefficient of restitution was changed by ball size. Minamoto et al. [12] collided two equivalent balls $25.4 \mathrm{~mm}, 13.5$ $\mathrm{mm}$ and $6.75 \mathrm{~mm}$ in diameter at high speed, 10 to 33 $\mathrm{m} / \mathrm{s}$. They clarified that the coefficient of restitution was changed by ball size and elastic wave propagation as the experimental and numerical results. How- ever, there is no precise report about that of two equivalent balls impact at lower speed than $1.0 \mathrm{~m} / \mathrm{s}$.

In the present study, the rebound property of two equivalent balls at relative low speed is examined and the influences of heat treating of the balls, the impact speed and the ball size on the rebound property are discussed. The ball diameters are $13.5 \mathrm{~mm}, 25.4 \mathrm{~mm}, 31.8 \mathrm{~mm}$ and $44.5 \mathrm{~mm}$. The balls are made of high carbon chromium bearing steel. Some of them are in the state of factory shipment. The others are annealed. In the experiment, two balls are collided at 0.2 to $1.2 \mathrm{~m} / \mathrm{s}$ velocity by using the motion of pendulum. Moreover, static and dynamic compressive material tests for the balls are performed and the relationship of the yield stress and the strain rate are obtained. Considering the dependence of yield stress on strain rate, the finite element analysis is executed. In order to separate the contributions of impact velocity and ball diameter, the coefficient of restitution is expressed as an exponential function of impact velocity and then the relationship between coefficients of the function and ball diameter are obtained. Comparing the experimental and numerical results, the changes of the coefficient of restitution and the mechanical energy, the stress and the strain in the balls, and so on are discussed. 


\section{IMPACT TEST}

The ball diameters used by the experiment are $13.5 \mathrm{~mm}$ (17/32 in), $25.4 \mathrm{~mm}$ ( $1 \mathrm{in}), 31.8 \mathrm{~mm}$ (1$1 / 4 \mathrm{in})$ and $44.5 \mathrm{~mm}(1-3 / 4 \mathrm{in})$. The ball material is high carbon chromium bearing steel (JIS SUJ2). The impact tests were conducted for the balls in the state of factory shipment and the annealed balls. The balls in the state of factory shipment have been hardened by cold forming and surface quenching in the manufacturing process. The annealed balls are softened at $850^{\circ} \mathrm{C}$ in nitrogen gas. In the present paper, the ball in the state of factory shipment and the annealed ball are called balls A and B, respectively.

Figure 1 shows the experimental apparatus. Figures 1 (a) and 1(b) are the front and side views, respectively. A thread is glued on a ball. The two balls are hanged at the top plate with the thread in $\mathrm{V}$ form. The distance between the two balls is the same as the ball diameter. The two balls are in touch each other before experiment. The radius of pendulum $R$ is determined as shown Table 1 in order that the thread does not sag and the maximum impact velocity reaches about $1 \mathrm{~m} / \mathrm{s}$. The right and left balls are called ball 1 and 2, respectively. The ball 1 collides with the ball 2. $\theta_{0}$ is an angle of the raised ball 1 before the collision. $\theta_{1}$ and $\theta_{2}$ are angles of the rising balls 1 and 2 after the collision, respectively. These angles are measured with the protractors which

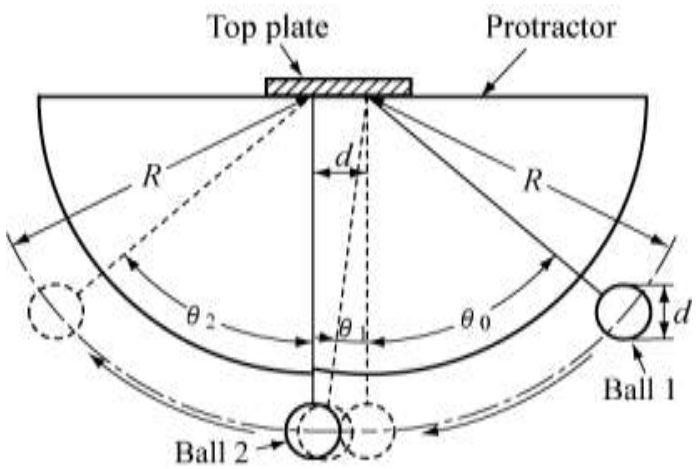

(a) Front view

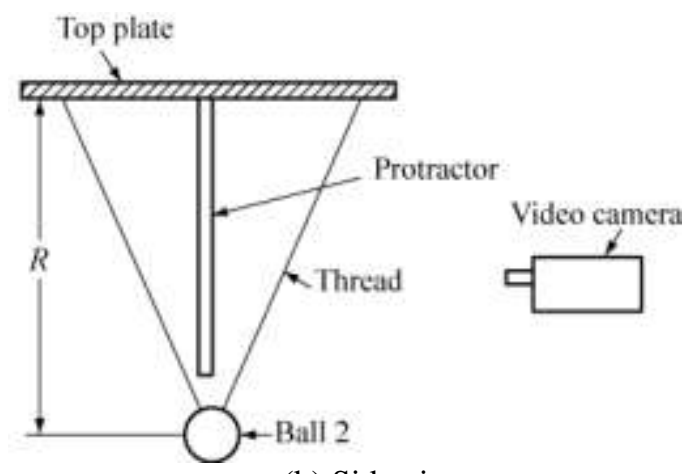

(b) Side view

Figure 1 pendulum impact apparatus center is the same location as the center of the pendulum and is fixed in a horizontal position. At the beginning, the ball 2 is set at rest. Next, the ball 1 is left by $\theta_{0}$, which is changed from $5^{\circ}$ to $35^{\circ}$ by $5^{\circ}$. And then the ball 1 is released to collide with the ball 2. $\theta_{1}$ and $\theta_{2}$ are measured four time at the same value of $\theta_{0}$. The relative averages of $\theta_{1}$ and $\theta_{2}$ are used to calculate velocities and so on described in the next section.

Table 1 Ball diameter and pendulum radius

\begin{tabular}{|c|c|}
\hline Ball diameter $d \mathrm{~mm}$ & Pendulum radius $R \mathrm{~mm}$ \\
\hline 13.5 & 0.40 \\
\hline 25.4 & 0.43 \\
\hline 31.8 & 0.43 \\
\hline 44.5 & 0.46 \\
\hline
\end{tabular}

\section{COEFFICIENT OF RESTITUTION} AND ENERGY LOSS

$v_{0}$ is the velocity of the ball 1 just before the collision, namely the impact velocity. $v_{1}$ and $v_{2}$ are the velocities of the balls 1 and 2 just after the collision, respectively. Neglecting the air resistance of the balls, the velocities $v_{0}, v_{1}$ and $v_{2}$ are given from $R$, $\theta_{0}, \theta_{1}$ and $\theta_{2}$ by the next equations:

$$
\begin{aligned}
& v_{0}=\sqrt{2 g R\left(1-\cos \theta_{0}\right)} \\
& v_{1}=\sqrt{2 g R\left(1-\cos \theta_{1}\right)} \\
& v_{2}=\sqrt{2 g R\left(1-\cos \theta_{2}\right)}
\end{aligned}
$$

where $g$ is the gravity acceleration. The coefficient of restitution $e$ is obtained by the next equation:

$$
e=\frac{v_{2}-v_{1}}{v_{0}}=\frac{\sqrt{1-\cos \theta_{2}}-\sqrt{1-\cos \theta_{1}}}{\sqrt{1-\cos \theta_{0}}}
$$

Because the two balls have the same mass, the following relationship is satisfied by the law of conservation of momentum:

$$
v_{0}=v_{1}+v_{2}
$$

Solving Eqns. (4) and (5) for $v_{1}$ and $v_{2}$, they are denoted by $v_{0}$ and $e$ as the next equations:

$$
v_{1}=\frac{1}{2}(1-e) v_{0}, \quad v_{2}=\frac{1}{2}(1+e) v_{0}
$$

If $e=1$, then $v_{1}=0, v_{2}=v_{0}$. After the collision, the ball 1 freezes. Instead, the ball 2 is accelerated by the same velocity as the impact velocity and rises by the same angle as the ball 1 is left. By the way, if $e<$ 1 , then $v_{1}>0, v_{2}<v_{0}$. The ball 1 has some velocity. Since the velocity of the ball 2 just after the collision is lower than $v_{0}, \theta_{2}$ is smaller than $\theta_{0}$.

$E_{0}$ is mechanical energy of the system before the collision. $\Delta E$ is energy loss of the system due to the collision. From the relations (1) (3), the 
ratio of the energy loss $\Delta E$ to the energy $E_{0}, \Delta E / E_{0}$, is represented by the next expression:

$$
\frac{\Delta E}{E_{0}}=\frac{\frac{1}{2} m v_{0}^{2}-\left(\frac{1}{2} m v_{1}^{2}+\frac{1}{2} m v_{2}^{2}\right)}{\frac{1}{2} m v_{0}^{2}}
$$

where, $m$ is the mass of the ball. Considering the dependence of the impact velocity, the coefficient of restitution is approximated by the next exponential function [6]:

$$
e=\exp \left(-\alpha v_{0}^{n}\right)
$$

Following Eqn. (8), Eqn. (7) is rewritten to the next equation:

$$
\frac{\Delta E}{E_{0}}=\frac{1}{2}\left\{1-\exp \left(-2 \alpha v_{0}^{n}\right)\right\}
$$

where, $\alpha$ and $n$ are coefficients determined from experimental results by the least square method. Considering the dependence of the coefficient of restitution $e$ on the ball size, the coefficients $\alpha$ and $n$ are related to the ball diameter.

\section{MATERIAL PROPERTY OF THE BALL}

In order to obtain the static relationship of stress and strain of the ball material, cylindrical specimens were made of the balls and had $8 \mathrm{~mm}$ in diameter and $20 \mathrm{~mm}$ long. The static compressive test is carried out with a universal testing machine. The strain rate is $0.05 / \mathrm{s}$. To obtain the dynamic relationship of stress and strain, the impact compressive test is performed by the Hopkinson bar method [13]. By using various sizes of specimen and loading conditions, the stress - strain curves for the different strain rates were measured [12][14].

\section{FINITE ELEMENT ANALYSIS}

Because the analytical object is axisymmetric, the axisymmetric numerical analysis of the two balls are executed based on the finite element method. Figure 2(a) shows the entire finite element subdivision and Fig. 2(b) is the wide view in the vicinity of contact part which area was meshed finely. The other area is meshed roughly. The elements are four-node axisymmetric elements. The material model includes the dependence of yield stress on strain rate. LS-DYNA [15][16] is a general dynamic non-linear analysis software based on the finite element method. The software is used for the analysis of the balls.

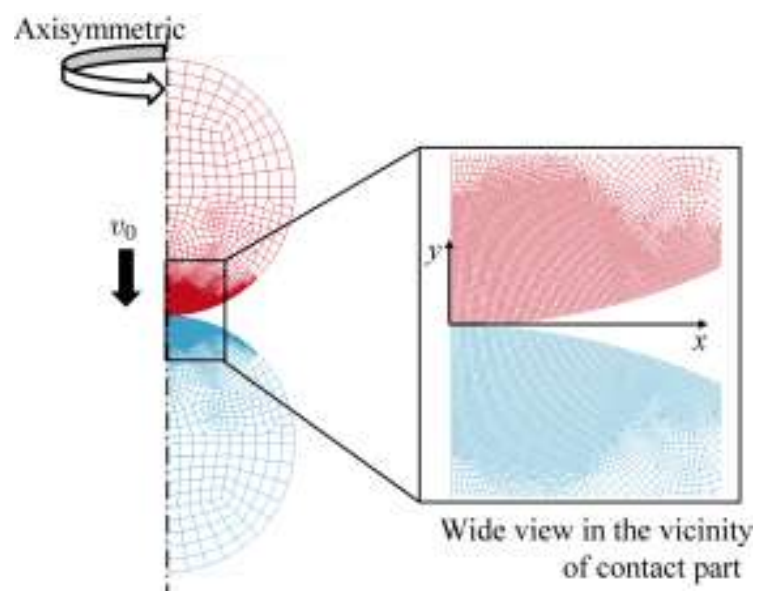

(a) Entire subdivision

(b) Fine meshed area

Figure 2 finite element subdivision

\section{RESULTS AND DISCUSSIONS}

\subsection{Material property of the ball}

Figure 3 shows the relations between the strain and its rate obtained from the six kinds of test condition for the impact compressive test. The vertical axis is strain rate and the horizontal axis is strain. When the strain rate increases, wave appears strongly in the measurement results. For each condition, the strain rate is regarded as constant shown as the broken line. They are 600/s, 1000/s, 1750/s, 2300/s, 2800/s and 4100/s, respectively.

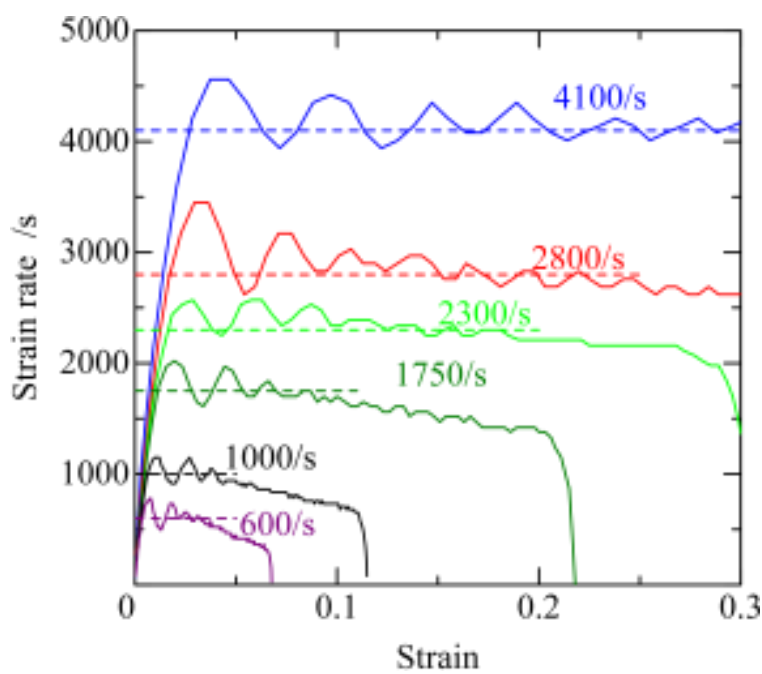

Figure 3 strain rate - strain relations of SUJ2

Figure 4 shows the relationship between the true stress and the true strain obtained by the static and dynamic compressive tests. The lines are corresponded to the strain rates shown in Fig. 3. The vertical axis is true stress and the horizontal axis is true strain. The compressive yield stress increases with the strain rate. Also, the true stress increases linearly after the yield. From the result of the static compressive test, Young's modulus is $202 \mathrm{GPa}$, the static compressive yield stress is $470 \mathrm{MPa}$. There are 
straight parts of the strain rates 600/s and 1000/s which are stead relatively after the yield. From their slopes, the tangent coefficient $E_{\mathrm{t}}$ is gotten by the least square method. As results, the slopes of the strain rates $600 / \mathrm{s}$ and $1000 / \mathrm{s}$ are $2.6 \mathrm{GPa}$ and 2.2 $\mathrm{GPa}$, respectively. Then it was determined that the tangent coefficient was the average of them, 2.4 GPa.

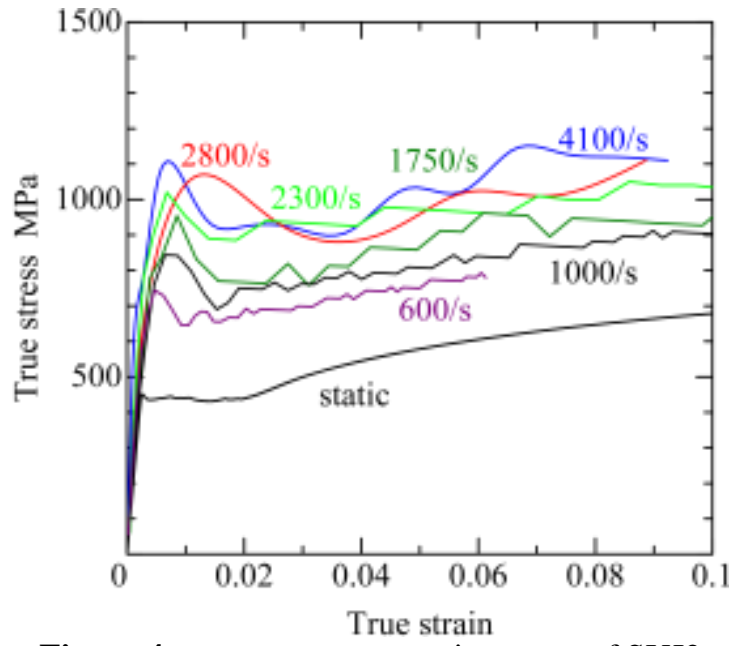

Figure 4 true stress - true strain curves of SUJ2

Figure 5 shows the relationship between the compressive yield stress and the strain rate obtained from Fig. 4. The vertical axis is compressive yield stress and the horizontal axis is strain rate. The static compressive yield stress $470 \mathrm{MPa}$ is plotted at $0.05 / \mathrm{s}$ strain rate. Because the results of the impact compressive test oscillate (Fig.3), the values of the strain rate and the compressive yield stress are read with increments of 50/s and $50 \mathrm{MPa}$, respectively. The broken line is a third order curve fitting the experimental data by the least square method. The compressive yield stress increases monotonically with the strain rate. When the strain rate is higher than $2500 / \mathrm{s}$, the compressive yield stress becomes constant $900 \mathrm{MPa}$.

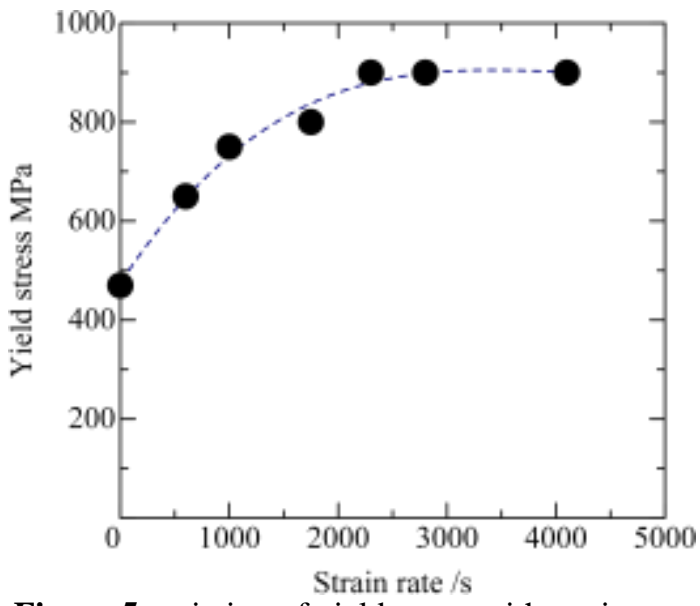

Figure 5 variation of yield stress with strain rate
The material properties are listed in Table 2. They and the relationship shown in Fig. 5 were inputted to the software, LS-DYNA as material data. The numerical analysis based on the finite element method was executed considering the dependence of yield stress on strain rate.

Table 2 Material properties of SUJ2 inputted in LSDYNA

\begin{tabular}{|l|c|}
\hline Density $\rho \mathrm{kg} / \mathrm{m}^{3}$ & 7850 \\
\hline Young's modulus $E \mathrm{GPa}$ & 202 \\
\hline Poisson's ratio $v$ & 0.3 \\
\hline Static yield stress $\sigma_{\mathrm{ys}} \mathrm{MPa}$ & 470 \\
\hline Tangent modulus $E_{\mathrm{t}} \mathrm{GPa}$ & 2.4 \\
\hline
\end{tabular}

\subsection{Experimental and analytical results for im- pact of the balls}

The respective averages of the experimental values of $\theta_{1}$ and $\theta_{2}$ obtained in the impact test are computed. $v_{1}$ and $v_{2}$ are calculated from the averages. Figure 6 shows the relationships between $v_{1}$ and $v_{0}$ and Fig. 7 shows one between $v_{2}$ and $v_{0}$. The horizontal axes in the both figures are $v_{0}$. The vertical axis in Fig. 6 is $v_{1}$ and one in Fig. 7 is $v_{2}$. Solid and empty marks indicate the results about the balls $\mathrm{A}$ and $\mathrm{B}$, respectively. The rectangles, circles, uppointing triangles and down-pointing triangles indicate $13.5 \mathrm{~mm}, 25.4 \mathrm{~mm}, 31.8 \mathrm{~mm}$ and $44.5 \mathrm{~mm}$ diameters, respectively. The broken lines are second order curves fitting the respective results by the least square method. In the case of $v_{0}=1.28 \mathrm{~m} / \mathrm{s}\left(\theta_{0}=\right.$ $\left.35^{\circ}\right)$, the thread separated with the ball $\mathrm{B}$ with a diameter of $44.5 \mathrm{~mm}$ during the experiment and then the result could not be obtained. $v_{1}$ of the ball $\mathrm{A}$ is almost $0 \mathrm{~m} / \mathrm{s}$ irrespective of $v_{0}$. By the way, $v_{1}$ of the ball B increases with $v_{0}$. The higher $v_{0}$, the higher $v_{1}$ of the ball B. $v_{2}$ of the ball $\mathrm{A}$ is almost the same

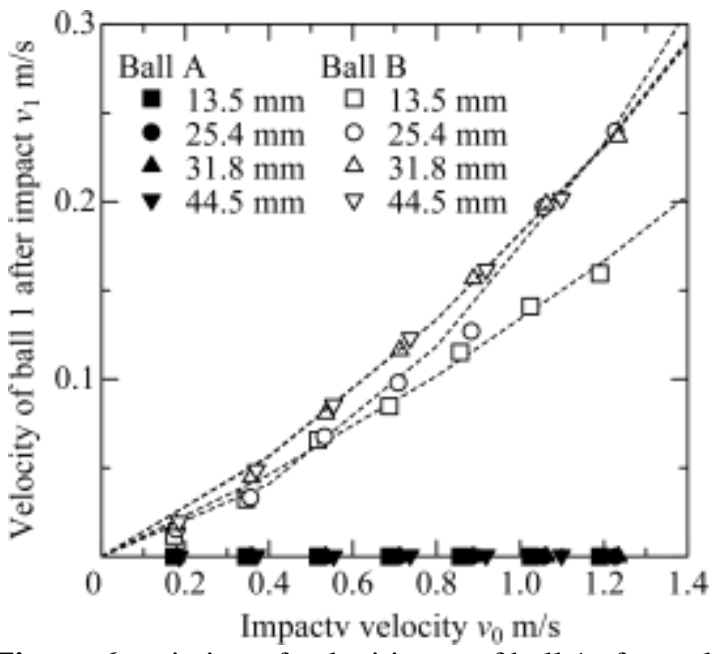

Figure 6 variation of velocities $v_{1}$ of ball 1 after collision with impact velocity $v_{0}$ 


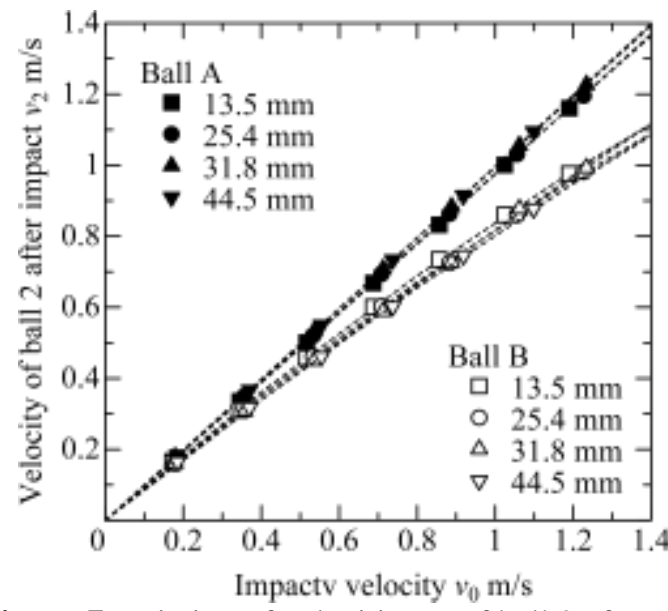

Figure 7 variation of velocities $v_{2}$ of ball 2 after collision with impact velocity $v_{0}$

value as $v_{0}$ irrespective of $v_{0}$. On the other hand, $v_{2}$ of the ball B is lower than $v_{0}$. The higher $v_{0}$, the larger difference between $v_{0}$ and $v_{2}$ of the ball $\mathrm{B}$. As discussed later, the tendency means that the ball $\mathrm{A}$ behaves when $e$ is near 1 and the ball $\mathrm{B}$ acts when $e$ is relatively smaller than 1 . For the relationship between $v_{1}, v_{2}$ and the ball diameter $d$, obviously, there is no much change of $v_{1}$ and $v_{2}$ of the ball A with $d$. However, the larger $d$, the higher $v_{1}$ of the ball $\mathrm{B}$ and the lower $v_{2}$ of the ball B.

Figure 8 shows the relationship between $e$ and $v_{0}$ calculated from Eqn. (4) for the balls $\mathrm{A}$ and $\mathrm{B}$. The vertical axis is $e$ and the horizontal axis is $v_{0}$. The broken lines are exponential curves of Eqn. (8) fitting the results of the ball $\mathrm{B}$ by the least square method. $e$ of the ball A is irrespective of $v_{0}$ and is about constant from 0.97 to 0.99 . It is not found the significant change of $e$ with $d$. Therefore, it is considered that the collision of ball A is almost perfectly elastic. On the other hand, it is seen that $e$ of the ball $\mathrm{B}$ tends to decrease with an increase in $v_{0}$. Also, it is turned out that there is a tendency that the larger $d$, the smaller $e$.

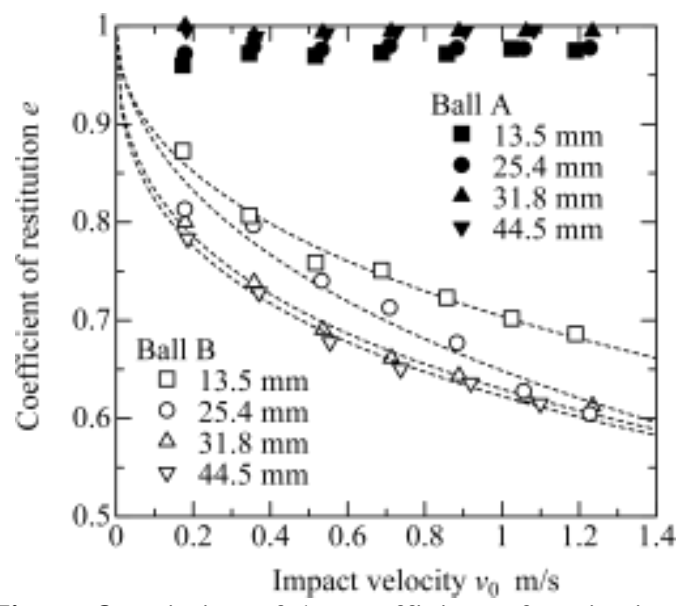

Figure 8 variation of the coefficient of restitution $e$ of balls $\mathrm{A}$ and $\mathrm{B}$ with impact velocity $v_{0}$
Figure 9 shows the relationship between $e$ and $v_{0}$ obtained by the finite element method (FEM) in addition to ones of the ball $\mathrm{B}$. The solid lines are exponential curves of Eqn. (8) fitting $e$ of FEM by the least square method. As well as the ball $\mathrm{B}, e$ obtained by FEM decreases with an increase of $v_{0}$. The larger $d$, the smaller $e$ obtained by FEM. In the case of the ball $\mathrm{B}, e$ of $d=31.8 \mathrm{~mm}$ and $44.5 \mathrm{~mm}$ are almost the same. $e$ of $d=13.5 \mathrm{~mm}$ and $25.4 \mathrm{~mm}$ are different from $e$ of $d=31.8 \mathrm{~mm}$ and $44.5 \mathrm{~mm}$. By the way, in the case of FEM, there is not a change of $e$ with $d$ when $v_{0}$ is equal to and lower than $0.3 \mathrm{~m} / \mathrm{s}$. And $e$ of $d=13.5 \mathrm{~mm}$ is larger than $e$ of the other ball diameters when $v_{0}$ is higher than $0.3 \mathrm{~m} / \mathrm{s}$. $e$ of $d$ $=25.4 \mathrm{~mm}, 31.8 \mathrm{~mm}$ and $44.5 \mathrm{~mm}$ are almost the same. As mentioned later, the cause is that the strain rate and the high stress region in the ball of $d=13.5$ $\mathrm{mm}$ are much larger than ones in the balls of the other diameter. The difference of $e$ with $d$ increases with an increment of $v_{0}$. In the case of $d=13.5 \mathrm{~mm}$ and $25.4 \mathrm{~mm}, e$ of the ball $\mathrm{B}$ and $e$ obtained by FEM are different. In the case of $d=31.8 \mathrm{~mm}$ and 44.5 $\mathrm{mm}$, they are much the same.

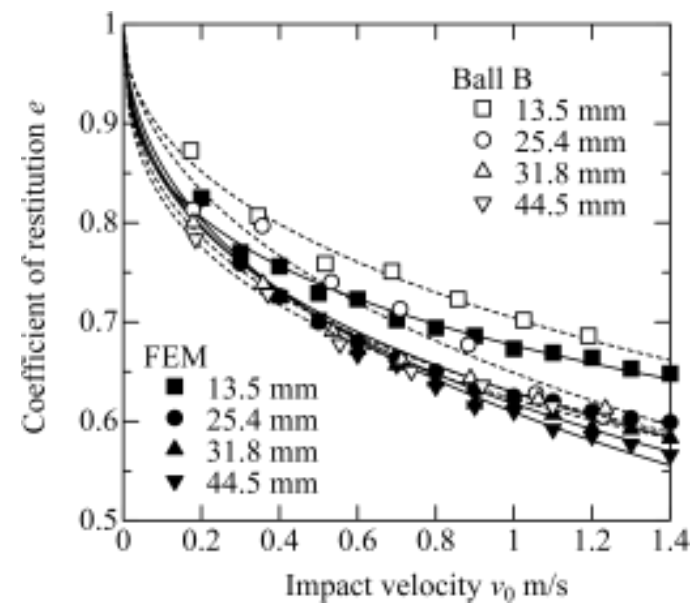

Figure 9 variation of the coefficient of restitution $e$ of ball B and FEM with impact velocity $v_{0}$

Figure 10 shows the relationship between $e$ and $d$ in the cases of $v_{0}=0.5 \mathrm{~m} / \mathrm{s}$ and $1.0 \mathrm{~m} / \mathrm{s}$. The values of the ball $\mathrm{B}$ are calculated by the linear interpolation for the experimental results in the vicinity of $v_{0}=0.5 \mathrm{~m} / \mathrm{s}$ and $1.0 \mathrm{~m} / \mathrm{s}$. The circular and triangular marks indicate $v_{0}=0.5 \mathrm{~m} / \mathrm{s}$ and $1.0 \mathrm{~m} / \mathrm{s}$, respectively. The empty and solid marks indicate the $e$ of the ball B and FEM, respectively. In the case of $v_{0}$ $=0.5 \mathrm{~m} / \mathrm{s}, e$ of the ball B decreases with an increment of $d$. However, $e$ obtained by FEM except $d=$ $13.5 \mathrm{~mm}$ is constant. When $v_{0}$ is lower than $0.5 \mathrm{~m} / \mathrm{s}$ and $d$ is larger than $25.4 \mathrm{~mm}$, the dependence of yield stress on the strain rate scarcely influence the results. Jackson et al. [9] compared several models to experimental results of aluminum oxide spheres impacting on a steel flat and pointed out that the 
similar reason. The tendency of $e$ obtained by FEM is different from $e$ of the ball $\mathrm{B}$. In the case of $v_{0}=$ $1.0 \mathrm{~m} / \mathrm{s}, e$ of the ball $\mathrm{B}$ and one of FEM decrease with an increment of $d$. The decrement rates reduce with increasing $d$. Also. $e$ obtained by FEM is smaller than $e$ of the ball $\mathrm{B}$.

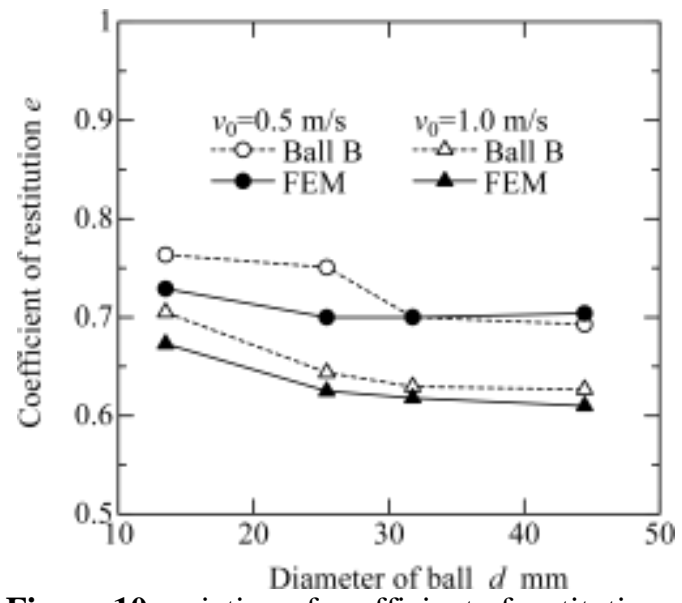

Figure 10 variation of coefficient of restitution $e$ with impact velocity $v_{0}$

Figure 11 shows $\Delta E / E_{0}$ calculated by Eqn. (7) for the ball B and FEM. The vertical axis is $\Delta E / E_{0}$ and the horizontal axis is $v_{0}$. The marks and lines identify the respective results as well as Fig. 9. $\Delta E / E_{0}$ increases with increasing $v_{0}$. When $v_{0}$ is lower than $0.2 \mathrm{~m} / \mathrm{s}, \Delta E / E_{0}$ increases rapidly with $v_{0}$. When $v_{0}$ is higher than $0.2 \mathrm{~m} / \mathrm{s}$, the increment rate of $\Delta E / E_{0}$ decreases little by little. $\Delta E / E_{0}$ of the ball B $13.5 \mathrm{~mm}$ in diameter is slightly smaller than ones of the ball $\mathrm{B}$ with other diameter, which are not much different together and increase with $d . \Delta E / E_{0}$ of $d=13.5 \mathrm{~mm}$ and one of $d=25.4 \mathrm{~mm}$ obtained by FEM are different from ones of the ball B. $\Delta E / E_{0}$ significantly changes with $v_{0}$ when $d$ is small.

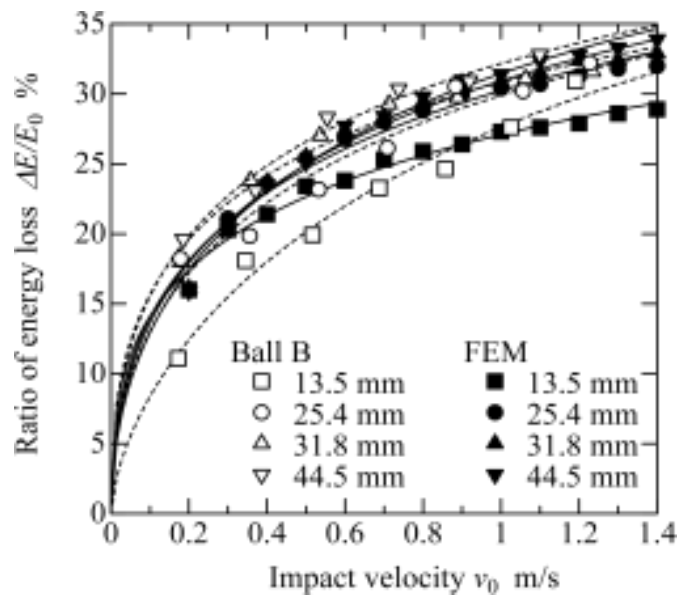

Figure 11 relationship of energy loss with impact velocity for ball B
The coefficients $\alpha$ and $n$ appear in the approximate expressions (8) of $e$ and (9) of $\Delta E / E_{0}$. Figure 12 shows the relationship between $\alpha$ and $d$. Also, Fig. 13 shows the relationship between $n$ and $d$. The circular and triangular marks indicate $e$ and $\Delta E / E_{0}$, respectively. The empty and solid marks indicate the results of ball B and FEM, respectively. In the case of ball $\mathrm{B}$, the reason for the difference between the results of $e$ and $\Delta E / E_{0}$ is that $v_{1}+v_{2}$ is not exactly equal to $v_{0}$. The average of $\left(v_{1}+v_{2}\right) / v_{0}$ is 0.990 and its range is 0.954 to 1.013 . On the other hand, in the case of FEM, the results of $e$ and $\Delta E / E_{0}$ are mostly coincident. In Fig. 12, $\alpha$ increases with $d$ and the increment rate decreases with $d$ little by little. For the ball $\mathrm{B}, \alpha$ of $\Delta E / E_{0}$ is larger than $\alpha$ of $e$ as a whole. For the results of FEM, $\alpha$ of $e$ and $\Delta E / E_{0}$ approximate to $\alpha$ of $\Delta E / E_{0}$ of the ball B. In Fig. 13, $n$ of the ball $\mathrm{B}$ does not change monotonically and has a tendency to decrease with $d$. However, $n$ of FEM increases monotonically and its tendency was different from $n$ of the ball B.

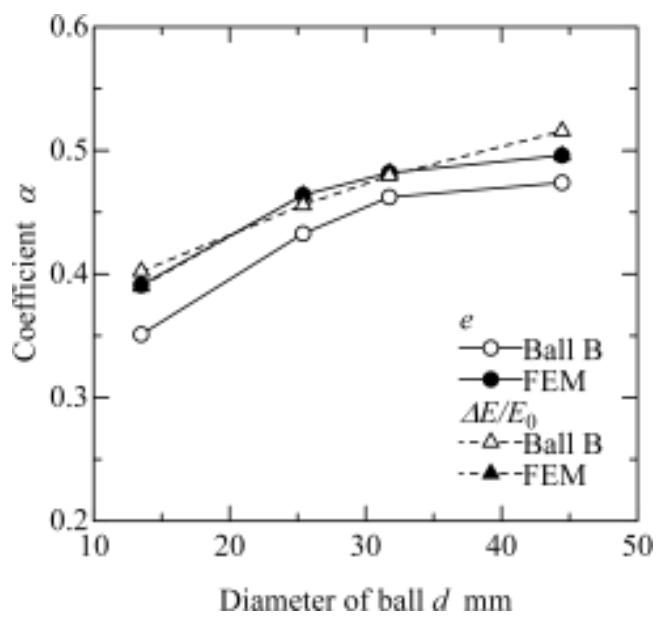

Figure 12 relationship of coefficient $\alpha$ and ball diameter $d$

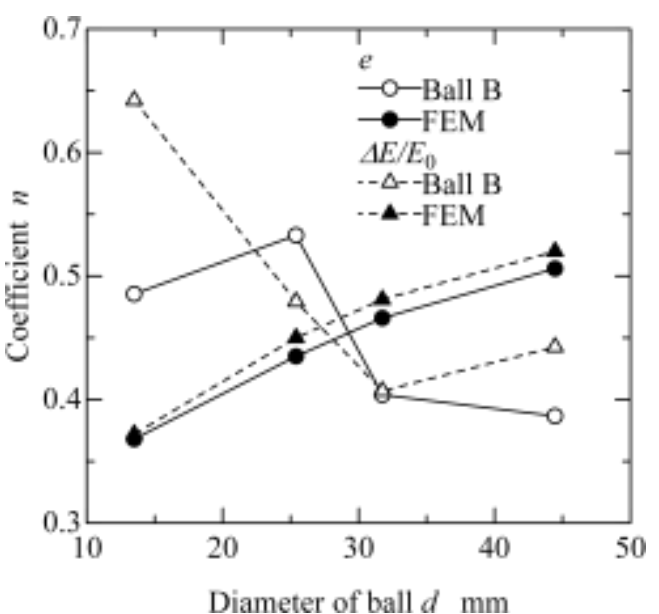

Figure 13 relationship of coefficient $n$ and ball diameter $d$ 
As the results of FEM, Table 3 is a list of the maximum equivalent strain rate $\dot{\varepsilon}$ max during the collision and the maximum equivalent stress $\sigma_{\max }$ when the ball deforms maximally. Also, Fig. 14 shows contour maps of equivalent stress $\sigma_{\max }$ in the vicinity of the contact part when the ball deforms maximally. The photo height is a fortieth of $d$. The arrows in the photos are locations where the maximum equivalent stress occurs. The larger $d$, the smaller $\dot{\varepsilon}$ max $\cdot \dot{\varepsilon}$ max of $d=25.4 \mathrm{~mm}$ is two thirds of one of $d=13.5 \mathrm{~mm}$ and $\dot{\varepsilon} \max$ of $d=44.5 \mathrm{~mm}$ is smaller than a half of one of $d=13.5 \mathrm{~mm}$. $\sigma_{\max }$ of $d=$ $13.5 \mathrm{~mm}$ is $10 \mathrm{MPa}$ larger than ones of the other diameters, which are about the same. Although $\sigma_{\max }$ does not change significantly, the incremental rate of yield stress decreases with a decrement of $\dot{\varepsilon} \max$. Consequently, it appears the decrement of $e$ with $d$.

Table 3 Equivalent strain rate and maximum equivalent stress $\left(v_{0}=1 \mathrm{~m} / \mathrm{s}\right)$

\begin{tabular}{|c|c|c|}
\hline Ball diameter $d \mathrm{~mm}$ & $\dot{\varepsilon} \max \times 10^{3} / \mathrm{s}$ & $\sigma_{\max } \mathrm{MPa}$ \\
\hline 13.5 & 30.8 & 1030 \\
\hline 25.4 & 20.5 & 930 \\
\hline 31.8 & 18.6 & 950 \\
\hline 44.5 & 13.6 & 960 \\
\hline
\end{tabular}

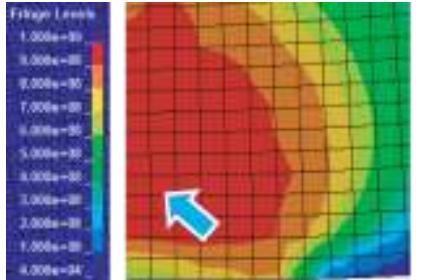

(a) $13.5 \mathrm{~mm}$
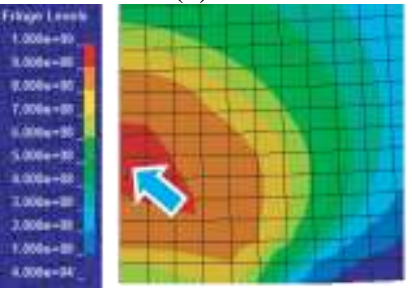

(c) $31.8 \mathrm{~mm}$

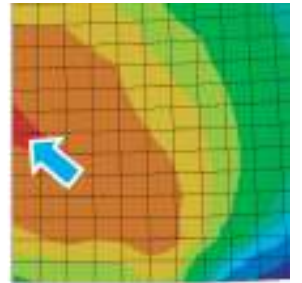

(b) $25.4 \mathrm{~mm}$

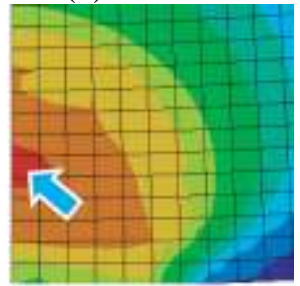

(d) $44.5 \mathrm{~mm}$
Figure 14 Contour maps of equivalent stress at maximum deformation $\left(v_{0}=1 \mathrm{~m} / \mathrm{s}\right)$

\section{CONCLUSION}

The Impact test of two equivalent balls was performed by utilizing the motion of pendulums. Some of them were in the state of factory shipment. The others were annealed. In addition, the numerical analysis based on the finite element method was executed considering the dependence of yield stress on strain rate as the material property of the annealed ball. As the results, the ball in the state of factory shipment behaves perfectly elastic collision and are independent of the impact velocity and the ball diameter. The coefficient of restitution of the annealed ball decreases with an increment of the impact velocity and a decrement of the ball diameter. The higher impact velocity, the larger decrement of the coefficient of restitution. The smaller ball diameter, the higher effect of ball size on the coefficient of restitution. The coefficients involved in the functions for the coefficient of restitution changed corresponding to the ball diameter. By using the relationship, it is possible to estimate results for an arbitrary ball diameter. In the numerical results by the finite element method, because the strain rate decreases with increasing the ball diameter, it is found that the coefficient of restitution decreases.

\section{REFERENCES}

[1] A. E. Love, A Treatise on the Mathematical Theory of Elasticity, 4th edition (Cambridge University Press, 1934).

[2] S. Timoshenko and N. Goodier, Theory of Elasticity, 3rd edition (McGRAW-HILL, New York, 1970).

[3] W. J. Stronge, Impact Mechanics (Cambridge University, 2004).

[4] T. Hong, J. Y. Ooi, B.A. Shaw, A numerical study of the residual stress pattern from single shot impacting on a metallic component, $A d$ vances in Engineering Software, 39, 2008, 743-756.

[5] C. Nouguier-Lehon, M. Zarwel, C. Diviani, D. Hertz, H. Zahouani, T. Hoc, Surface impact analysis in shot peening process, Wear, 302, 2013, 1058-1063.

[6] M. H. A. Hassan, Z. Taha, Finite Element Analysis of Soccer Heading, Procedia Engineering, 112, 2015, 46-51.

[7] T. Y. Pang, A. Subic, M. Takla, Finite element analysis of impact between cricket ball and cantilever beam, Procedia Engineering, 13, 2011, 258-264.

[8] M. Batista, G. Zovak, A RESTITUTION MODEL OF TWO-CAR COLLINEAR COLLISIONS, Promet - Traffic \& Transportation, 19(1), 2007, 1-6.

[9] R. L. Jackson, I. Green, D. B. Marghitu, Predicting the coefficient of restitution of impacting elastic-perfectly plastic spheres, Nonlinear Dynamics, 60, 2010, 217-229.

[10] M. Ahmad, K. A. Ismail, F. Mat, IMPACT MODELS AND COEFFICIENT OF RESTITUTION: A REVIEW, ARPN Journal of Engineering and Applied Sciences, 11(10), 2016, 6549-6555.

[11] A. Aryaei, K. Hashemnia, K. Jafarpur, Experimetal and Numerical Study of Ball Size Effect on Restitution Coefficient in Low Velocity Impacts, International Journal of Impact Engineering, 37, 2010, 1037-1044. 
[12] H. Minamoto, K. Onodera, S. Kawamura, E. Inamura, The Effect of Ball Size on the Rebound Property and Elastic Wave Propagation in Two-Sphere Impact, Proc. 20th International Congress on Sound and Vibration, Bangkok, Thailand, 2013, 419-426.

[13] B. Hopkinson, A Method of Measuring the Pressure in the Deformation of High Explosives or by the Impact of Bullets, Phil. Trans. Royal Soc., A213, 1914, 437-452.

[14] H. Minamoto, Elasto/Visco-Plastic Impact of Two Equivalent Spheres Made of SUJ2, Transactions of the Japan Society of Mechanical Engineers, Series C, 71 (701), 2005, 5457 (in Japanese).

[15] LS-DYNA KEYWORD USER'S MANUAL, Version 971 (Livermore Software Technology Corporation, 2007).

[16] LS-DYNA THEORY MANUAL (Livermore Software Technology Corporation, 2006).

[17] The Japan Society of Mechanical Engineers (Ed.), JSME Mechanical Engineers' Handbook, 4th revised edition (The Japan Society of Mechanical Engineers, 1967) (in Japanese). 